\title{
Resection Process Map: A novel dynamic simulation system for pulmonary resection
}

Junko Tokuno, MD, ${ }^{a}$ Toyofumi F. Chen-Yoshikawa, MD, PhD, ${ }^{a}$ Megumi Nakao, $\mathrm{PhD},{ }^{b}$

Tetsuya Matsuda, MD, $\mathrm{PhD},{ }^{\mathrm{b}}$ and Hiroshi Date, $\mathrm{MD}, \mathrm{PhD}^{\mathrm{a}}$

\section{ABSTRACT}

Objective: Use of 3-dimensional computed tomography for preoperative and intraoperative simulation has been introduced in the field of thoracic surgery. However, 3-dimensional computed tomography provides only static simulation, which is a significant limitation of surgical simulation. Dynamic simulation, reflecting the intraoperative deformation of the lung, has not been developed. The aim of this study was to develop a novel simulation system that generates dynamic images based on patient-specific computed tomography data.

Methods: We developed an original software, the Resection Process Map, for anatomic pulmonary resection. The Resection Process Map semi-automatically generates virtual dynamic images based on patient-specific computed tomography data. We retrospectively evaluated its accuracy in 18 representative cases by comparing the virtual dynamic images with the actual surgical images.

Results: In this study, 9 patients who underwent lobectomy and 9 patients who underwent segmentectomy were included. For each case, a virtual dynamic image was successfully generated semi-automatically by the Resection Process Map. The Resection Process Map accurately delineated 98.6\% of vessel branches and all the bronchi. The median time required to obtain the images was $121.3 \mathrm{sec}-$ onds.

Conclusions: We successfully developed a novel dynamic simulation system, the Resection Process Map, for anatomic pulmonary resection. (J Thorac Cardiovasc Surg 2020;159:1130-8)

This novel simulation system generates virtual dy-
namic images based on patient-specific CT data.
Central Message
We successfully developed a novel simulation
system that generates virtual dynamic images
based on patient-specific CT data for anatomic
pulmonary resection.
Perspective
We successfully developed a novel simulation
system that generates virtual dynamic images
based on patient-specific CT data for anatomic
pulmonary resection. We retrospectively as-
sessed the usefulness of this system. Then, we
prospectively evaluated its efficacy as a preop-
erative/intraoperative simulation and an educa-
tive tool for future study.
See Commentaries on pages 1139 and
1141 .
1140.

Video-assisted thoracoscopic surgery (VATS) has become a standard approach for the treatment of lung cancer, metastatic lung tumors, and other benign nodules. However, the

From the ${ }^{\mathrm{a}}$ Department of Thoracic Surgery, Graduate School of Medicine, and ${ }^{\mathrm{b}}$ Graduate School of Informatics, Kyoto University, Kyoto, Japan.

This work was supported by a grant from the Japan Agency for Medical Research and Development (Acceleration Transformative Research for Medical Innovation, No. 17im0210215).

Read at the 99th Annual Meeting of The American Association for Thoracic Surgery, Toronto, Ontario, Canada, May 4-7, 2019.

Received for publication April 29, 2019; revisions received July 24, 2019; accepted for publication July 25, 2019; available ahead of print Oct 9, 2019.

Address for reprints: Toyofumi F. Chen-Yoshikawa, MD, PhD, Department of Thoracic Surgery, Kyoto University Hospital, 54 Shogoin-Kawahara-cho, Sakyoku, Kyoto 606-8507, Japan (E-mail: fengshic@kuhp.kyoto-u.ac.jp).

0022-5223

Copyright (C) 2019 by The American Association for Thoracic Surgery. Published by Elsevier Inc. This is an open access article under the CC BY-NC-ND license (http:// creativecommons.org/licenses/by-nc-nd/4.0/).

https://doi.org/10.1016/j.jtcvs.2019.07.136 minimally invasive nature, limited field of vision, and lack of tactile experience of VATS are unresolved significant limitations. A comprehension of the intraoperative deformation of the lungs is frequently challenging even for experienced surgeons. Considering these difficulties, the preoperative evaluation of anatomy by using images is crucial for VATS.

Scanning this QR code will take you to the article title page to access supplementary information. To view the AATS Annual Meeting Webcast, see the URL next to the webcast thumbnail.

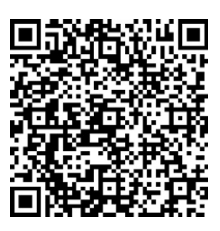



Abbreviations and Acronyms
$\mathrm{CT}=$ computed tomography
$\mathrm{IQR}=$ interquartile range
PA = pulmonary artery
$\mathrm{PV}=$ pulmonary vein
RPM $=$ Resection Process Map
STL = stereolithography
3D $=3$-dimensional
VATS $=$ video-assisted thoracoscopic surgery

Three-dimensional (3D) computed tomography (CT) imaging is a recently developed technology that has been shown to be useful in thoracic surgery. ${ }^{1-7}$ Some newly developed systems allow thoracic surgeons to construct 3D images without the need to consult a radiologist or technician, which represents a considerable advantage. ${ }^{1,2,6}$ Nonetheless, surgeons still need to rely on their experience because these systems provide only static images.

Regarding virtual dynamic images, a few recent reports have described the innovative use of virtual reality simulation as a training tool. ${ }^{7,8}$ Although these are useful tools for surgical training, their clinical application for surgical planning is limited, because individual patient-specific CT data cannot be incorporated in the generated image. In this context, there is an as-yet unmet need to develop a novel simulation system that generates a virtual dynamic image based on patient-specific CT data and simulates the intraoperative anatomic changes, such as those by a traction of surrounding lung parenchyma for a better exposure of the surgical field.

We previously reported on the Resection Process Map (RPM) as an intraoperative cutting guide for soft tissue resection procedures. ${ }^{9,10}$ The RPM, which is directly generated from patient-specific CT data, shows a variety of curved resection paths and provides a high-quality visual map for preoperative/intraoperative use. We designed a set of algorithms to provide a semiautomatic system framework tuned for planning and developed a novel system to generate virtual images at high speed, which can also be manipulated without difficulty by thoracic surgeons (Figure 1). In the current study, we aimed to develop the RPM for anatomic pulmonary resection and evaluate its clinical applicability.

\section{MATERIALS AND METHODS}

\section{Development and Function of the Resection Process} Map for Pulmonary Resection

We developed the RPM and applied the system to liver resection., ${ }^{9,10}$ In that version, to enable surgeons to simulate maneuvers, such as holding, grasping, and traction, using the system interactively in real-time, we used finite-element modeling, which can perform fast computation. ${ }^{11,12}$

In this study, we sought to further develop the RPM to improve its clinical utility and to apply it to pulmonary resection. In particular, we sought to reduce the data volume and to render the images more amenable to manipulation in the RPM. We attempted to use the surface data from patient-specific 3D reconstruction (based on CT data) to generate a virtual

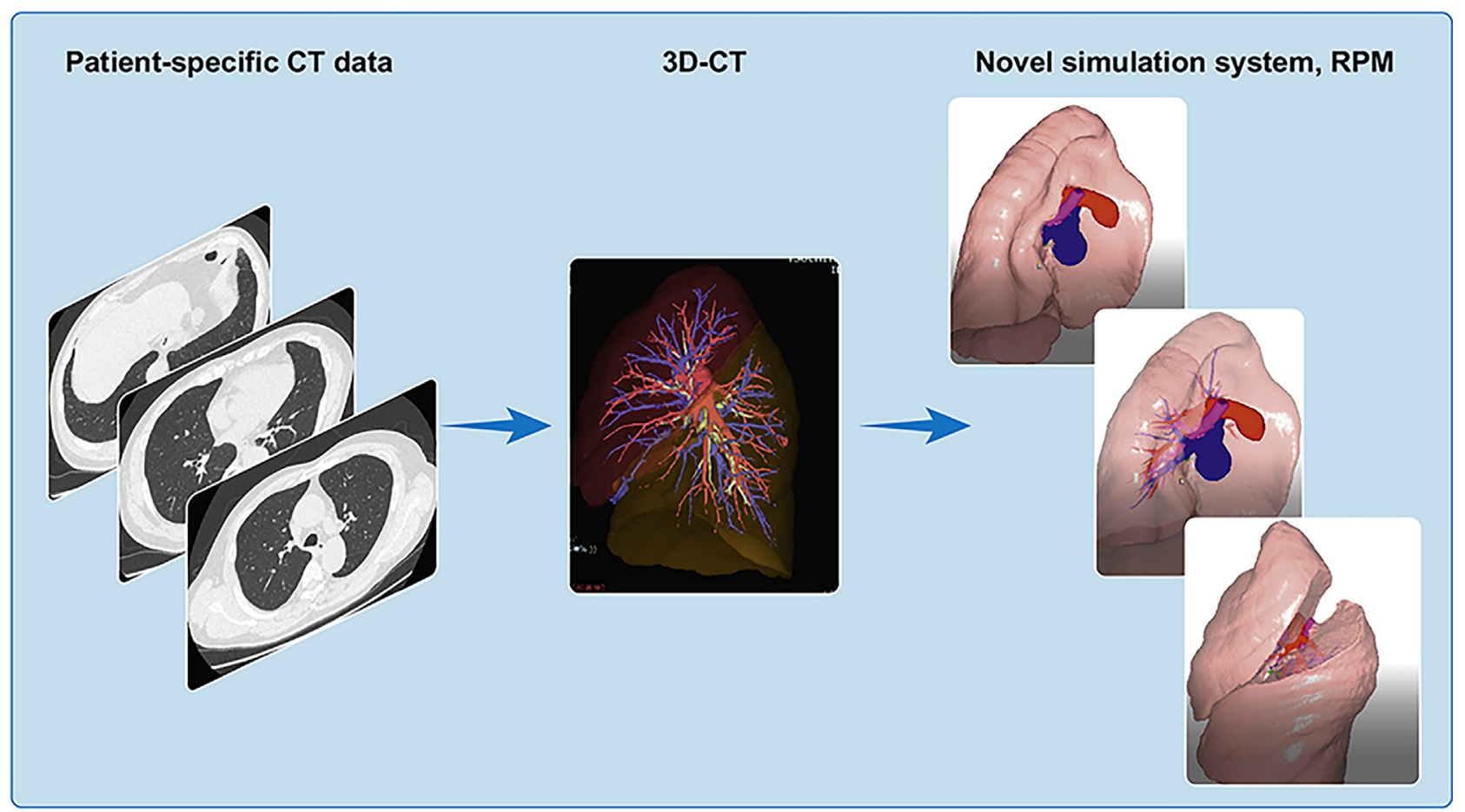

FIGURE 1. Design of the novel simulation system. A virtual dynamic image is generated from 3D-CT based on patient-specific CT data. CT, Computed tomography; RPM, Resection Process Map. 

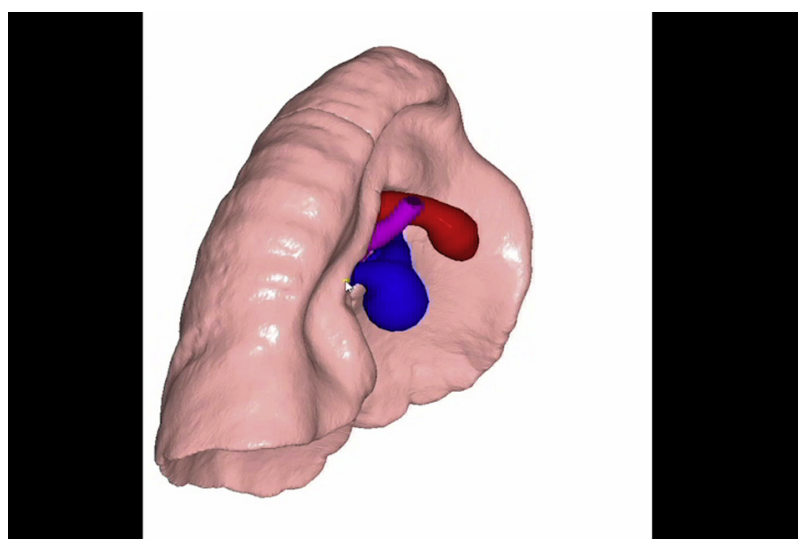

VIDEO 1. Original function of the RPM compared with conventional 3DCT. RPM showed the following maneuvers: to tract the lung arbitrarily, to visualize vessels and bronchi running inside the lung, and to open the fissure. Video available at: https://www.jtcvs.org/article/S0022-5223(19) 31769-6/fulltext.

dynamic image and added centerline detection of vessels and bronchi, which is widely adapted to model 3D images from 2-dimensional tubular or linear structures, such as vessels and bronchus. ${ }^{13-15}$ whereas in the previous version, we used Digital Imaging and Communications in Medicine data. ${ }^{9,10}$

We tried to accurately delineate the hilum structure and the interlobar view in the RPM considering the features of pulmonary resection and incorporated the following functionalities instead of the static image provided by conventional 3D-CT rotating (Video 1). It is manipulated by a mouse device and has some unique functions, including the following: (1) to provide views of the 3D model from arbitrary directions; (2) to tract the lung arbitrarily; (3) to visualize the vascular structures and bronchi through the lung parenchyma; (4) to mimic the deformation of vessels and bronchi upon deformation of the lung; and (5) to replicate changes in the RPM in response to each manipulation without delay (Video 1). During deformation in the RPM, hilar structures, such as the main pulmonary artery (PA), left atrium, and main bronchus, were fixed. With regard to elastic modulus parameters or Young's modulus of the lung parenchyma, they were set as $10 \mathrm{kPa}^{16}$

\section{How to Obtain the Virtual Dynamic Image}

Each patient underwent contrast-enhanced CT preoperatively. The 3D image analysis system (SYNAPSE VINCENT, Fuji Film Co, Ltd, Tokyo, Japan) at the Department of Thoracic Surgery at the Kyoto University was used to obtain 3D images of the pulmonary vessels and the tracheobronchial tree; subsequently, lobar and sublobar segmentation were performed using the system software. The process required only 1 phase contrast-enhanced CT. After manually designating the lobe or segment to be resected by the surgeon, the system extracted information pertaining to the pulmonary structures and displayed 3D static images.

The surface data of 3D images were extracted in the stereolithography (STL) file format from the SYNPSE VINCENT. Surface data of the lung (lobes and segments), vessels, bronchi, and tumor were separately saved.

This system was operated using another single computer terminal. A computer with graphic processing units (Intel Corei7 3.60 GHz (Intel Corporation, San Francisco, Calif); Memory: 32.0 GB; GPU: NVIDIA GeForce GTX 1070 (NVIDIA Corporation, Santa Clara, Calif) was used to convert the data.

In the next step, the STL data of the vessels (PA and pulmonary vein $[\mathrm{PV}])$ and the bronchi were input into a centerline detecting software (a supplemental application). During this step, the only action required was to specify the most proximal point. After setting the most proximal point, the software semi-automatically detected the centerline of the vessels and the bronchi.

Last, the surface data of the lung and tumor and centerline data of vessels and bronchi were input into the RPM, which generated a clear virtual image (Figure 2). By all these steps, the system allows the surgeon to obtain a virtual image semi-automatically (Video 2 ).

\section{Analysis}

First, the accuracy in the RPM was postoperatively assessed by evaluating how many branches of PA, PV, and bronchi that were sacrificed during the surgery, and this was evaluated by 2 surgeons (J.T. and T.F.C.-Y.). Second, the time required for each step after analysis by the SYNAPSE VINCENT (ie, the time required for detection of centerlines and the time required for RPM processing) was measured to evaluate how quickly the virtual images were obtained. Third, to see how smoothly the virtual image

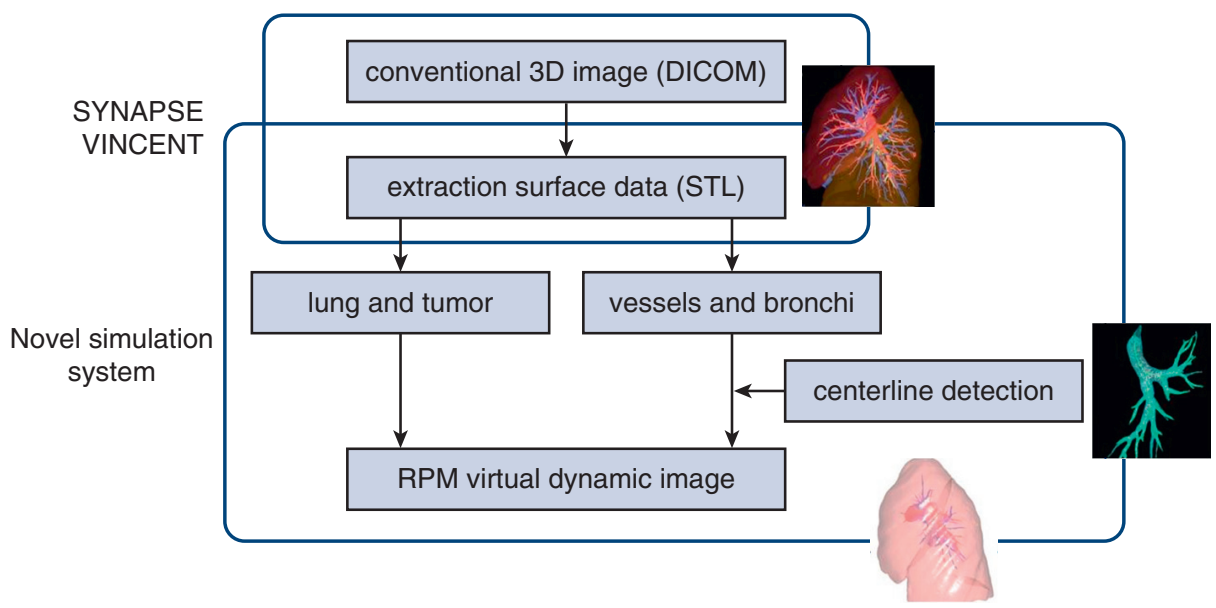

FIGURE 2. Surface data were extracted from conventional 3D image in SYNAPSE VINCENT (Fuji Film Co, Ltd, Tokyo, Japan). Surface data of the lung and tumor were input into the RPM, and surface data of vessels and bronchi were input to the RPM after centerline detection process. DICOM, Digital Imaging and Communications in Medicine; STL, stereolithography; RPM, Resection Process Map. 


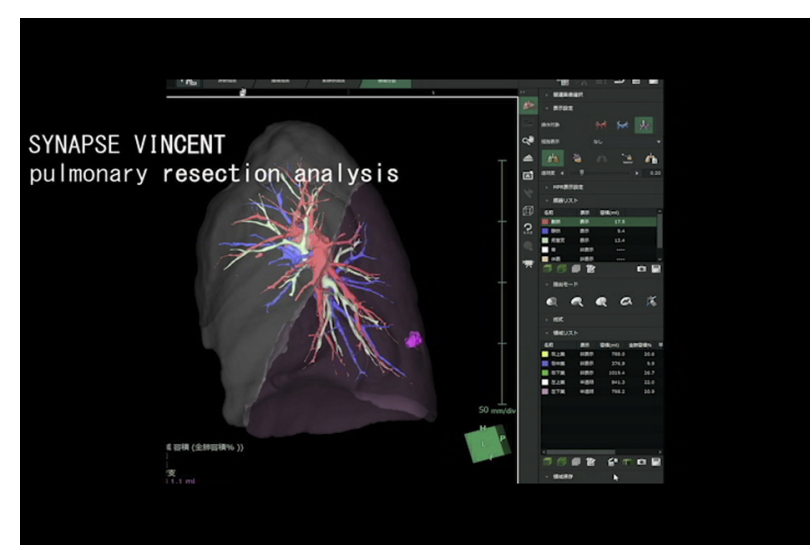

VIDEO 2. Three steps to generate virtual dynamic image. The first step is to transform the Digital Imaging and Communications in Medicine data to STL format. The second step is to perform centerline detection, which is needed for tubular construction. These 2 steps are necessary to reduce the data volume and to make the system quicker. The last step is to put the all data into the RPM software. Video available at: https://www.jtcvs.org/ article/S0022-5223(19)31769-6/fulltext.

was manipulated, the calculation time was recorded in each case. The calculation time is the time needed to show virtual image dynamic. Eighteen patients who underwent VATS anatomic lung resection between November 2017 and May 2018 were enrolled.

All participants provided informed consent before their enrollment and data collection. The analyses were approved by the institutional review board of the Kyoto University Hospital. All statistical analyses were performed using GraphPad software (Prism 7 version) (GraphPad Software, San Diego, Calif).

\section{RESULTS}

Thirteen men and 5 women (median age, 61.5 years; interquartile range [IQR], the difference between 75 th and 25th percentiles: 53-66.5) were included. Lung cancer was the most common indication for surgery $(\mathrm{n}=15)$. Lobectomy was performed in 9 patients, and segmentectomy

\section{TABLE 1. Patient characteristics in retrospective study}

\begin{tabular}{lc}
\hline & Median (IQR), cases \\
\hline Age, y & $61.5(53-66.5)$ \\
Sex, male/female & $13 / 5$ \\
Indication & \\
$\quad$ Primary lung cancer & 15 \\
$\quad$ Metastatic tumor & 2 \\
$\quad$ Arteriovenous malformation & 1 \\
Approach & \\
VATS & 18 \\
$\quad$ open & 0 \\
Operation & \\
Lobectomy & 9 \\
Segmentectomy & 9 \\
\hline
\end{tabular}

$I Q R$, Interquartile range; VATS, video-assisted thoracic surgery.
TABLE 2. Surgical procedures, indication, and time required for detecting centerline and building the virtual image and the calculation time in the resection process map

\begin{tabular}{|c|c|c|c|c|c|}
\hline $\begin{array}{l}\text { Case } \\
\text { no. }\end{array}$ & $\begin{array}{c}\text { Surgical } \\
\text { procedures }\end{array}$ & $\begin{array}{c}\text { Centerline } \\
\text { (s) }\end{array}$ & $\begin{array}{c}\text { RPM } \\
\text { (s) }\end{array}$ & $\begin{array}{c}\text { Total } \\
\text { (s) }\end{array}$ & $\begin{array}{c}\text { Calculation } \\
\text { time (ms) }\end{array}$ \\
\hline 1 & Lobectomy (RU) & 65.9 & 36.8 & 102.7 & 16.5 \\
\hline 2 & Lobectomy (RU) & 65.8 & 47.5 & 113.3 & 14.8 \\
\hline 3 & Lobectomy (RU) & 69.7 & 30.1 & 99.7 & 17.7 \\
\hline 4 & Lobectomy (RM) & 130.3 & 42.6 & 172.9 & 15.3 \\
\hline 5 & Lobectomy (RL) & 97.5 & 23.9 & 121.4 & 11.5 \\
\hline 6 & Lobectomy (RL) & 97.7 & 45.1 & 142.8 & 16.0 \\
\hline 7 & Lobectomy (LU) & 87.7 & 14.8 & 102.5 & 25.5 \\
\hline 8 & Lobectomy (LL) & 97.5 & 23.9 & 121.4 & 14.6 \\
\hline 9 & Lobectomy (LL) & 74.7 & 22.7 & 97.4 & 19.8 \\
\hline 10 & $\begin{array}{l}\text { Segmentectomy } \\
\quad \text { (Rt S2) }\end{array}$ & 105.8 & 29.2 & 135.0 & 14.3 \\
\hline 11 & $\begin{array}{l}\text { Segmentectomy } \\
\quad \text { (Rt S6) }\end{array}$ & 77.7 & 42.7 & 120.4 & 14.7 \\
\hline 12 & $\begin{array}{l}\text { Segmentectomy } \\
\quad \text { (Rt S8) }\end{array}$ & 113.8 & 81.3 & 195.1 & 6.8 \\
\hline 13 & $\begin{array}{l}\text { Segmentectomy } \\
\quad \text { (Lt S1-3) }\end{array}$ & 105.9 & 21.6 & 127.5 & 15.2 \\
\hline 14 & $\begin{array}{l}\text { Segmentectomy } \\
\quad(\mathrm{Lt} \mathrm{S} 1+2)\end{array}$ & 81.9 & 12.1 & 94.0 & 20.1 \\
\hline 15 & $\begin{array}{l}\text { Segmentectomy } \\
\quad(\text { Lt S3) }\end{array}$ & 113.1 & 12.1 & 125.2 & 18.4 \\
\hline 16 & $\begin{array}{l}\text { Segmentectomy } \\
\quad \text { (Lt S6) }\end{array}$ & 105.8 & 29.2 & 135 & 16.5 \\
\hline 17 & $\begin{array}{l}\text { Segmentectomy } \\
\quad(\text { Lt S8+9) }\end{array}$ & 95.5 & 18.1 & 113.6 & 13.7 \\
\hline 18 & $\begin{array}{l}\text { Segmentectomy } \\
\quad(\text { Lt S9+10) }\end{array}$ & 91.8 & 29.4 & 121.2 & 14.0 \\
\hline
\end{tabular}

$R P M$, Resection Process Map; $R U$, right upper lobe; $R M$, right middle lobe; $R L$, right lower lobe; $L U$, left upper lobe; $L L$, left lower lobe; $R t$, right; $L t$, left.

was performed in 9 patients (Table 1). The resected lobes and segments are listed in Table 2.

Regarding the accuracy of the virtual dynamic images, the RPM described all the vascular branches, which were identified on 3D reconstruction by the SYNAPSE VINCENT. Among the 18 cases, 39 branches of PA were sacrificed, but no branch was missed in the RPM. For the $\mathrm{PV}$, only 1 vein, of 18 , in a left $\mathrm{S} 1+2$ segmentectomy case was missed because the vein was not identified by the SYNAPSE VINCENT. There were no missed bronchi describing 18 of 18 branches. The accuracy calculated by their sum was $98.6 \%$.

The total time required in the steps to generate virtual images ranged from 97.4 to 195.1 seconds (median, 121.3; IQR, 102.6-135.0 seconds) (Table 2). There was no significant difference between the time required to generate 


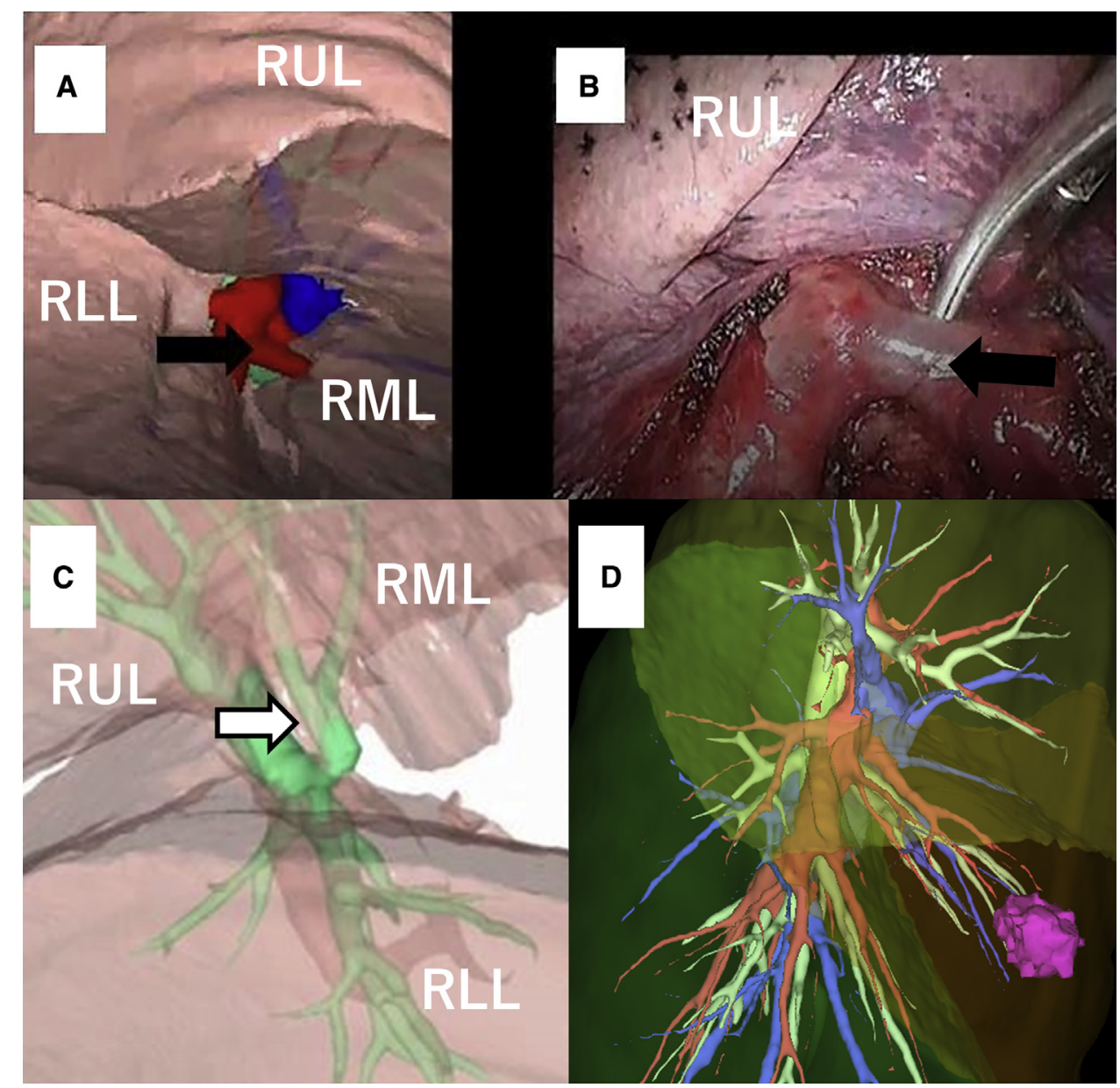

FIGURE 3. Representative case of right middle lobectomy comparing virtual image and surgical video. Virtual image was replicated by referring to surgical video. A, Snapshot of a dynamic image in the RPM during dissection of fissure. The black arrow in A indicates A4, which is under dissection in B. B, Screen grab from the surgical video during dissection of fissure. C, Snapshot of a dynamic image in the RPM during encircling bronchus. The white arrow in C indicates $\mathrm{B} 4+5$. D, Conventional 3D-CT. RUL, Right upper lobe; $R L L$, right lower lobe; $R M L$, right middle lobe.

the virtual image for lobectomy and segmentectomy (median, 113.3; IQR, 101.1-132.1 seconds and 125.2; IQR, 117.0-135.0 seconds, respectively; $P=.108$; Mann-Whitney $U$ test). The calculation time ranged from 6.8 to $20.1 \mathrm{~ms}$ (median, 15.3 ; IQR, $14.8-17.4 \mathrm{~ms}$ ) to show virtual dynamic image in the RPM. This means the virtual images could show approximately 65 frames in 1 second in the median.

We share 2 representative cases of patients who underwent anatomic VATS pulmonary resection (right middle lobectomy and left S3 segmentectomy). Both procedures were 3-port VATS. The case numbers correspond to those listed in Table 2. We compared the virtual images and actual surgical images of these 3 cases (Figures 2 and 3 and Videos 3 and 4).

\section{Case 4}

Case 4 underwent right middle lobectomy. First, we dissected the hilum to detect the middle PV. Then, we opened the major fissure and dissected the interlobar PA. In this case, there were $2 \mathrm{PA}$ branches that perfused into the middle lobe. We identified and cut the A4, which had branched out from the trunk peripherally before A5. Because the A4 ran along $\mathrm{B} 4+5$, we cut the $\mathrm{B} 4+5$ before A5 for convenience. Figure 2 shows the actual intraoperative image and the RPM image. The gauge of $\mathrm{PA}$ and $\mathrm{B} 4+5$ was well delineated in the RPM (Figure 3 and Video 3).

\section{Case 15}

Case 15 underwent left S3 segmentectomy. After dissecting the hilum, V3 was sacrificed while preserving the V4+5. After cutting V3, the A3 and the mediastinal A $4+5$ were dissected. Figure 3 shows the comparison of surgical video and the RPM image. In the RPM, the course of the PV and PA, including a tiny branch of A3, is well described (Figure 4 and Video 4). 


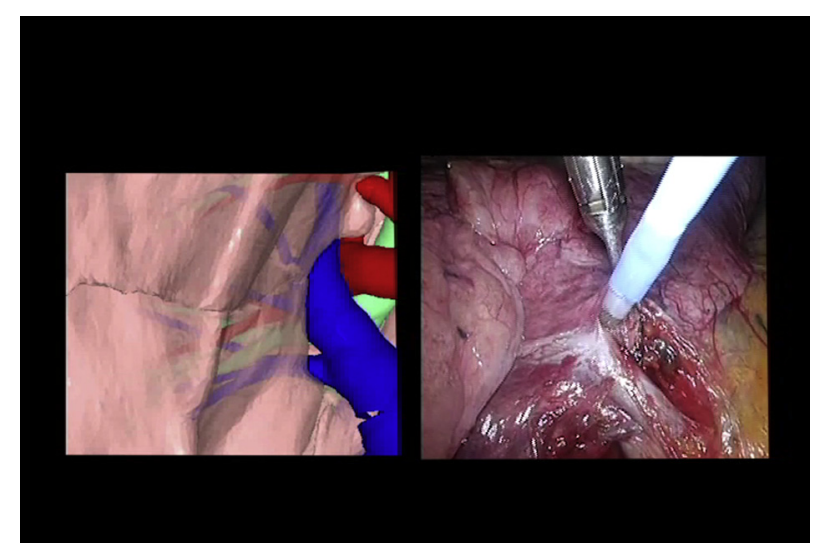

VIDEO 3. Comparison of the virtual dynamic image and the surgical video in a case undergoing right middle lobectomy. Virtual image is on the left side, surgical video is on the right side. PV is in blue, PA is in red, and bronchi is in purple. First, showing a scene of hilar PV. It was described similarly. Then, after opening the fissure, the PA was identified. A4 and 5 were also described accurately. After cutting the PA, we encircled B4+5 and divided it. Video available at: https://www.jtcvs.org/article/ S0022-5223(19)31769-6/fulltext.

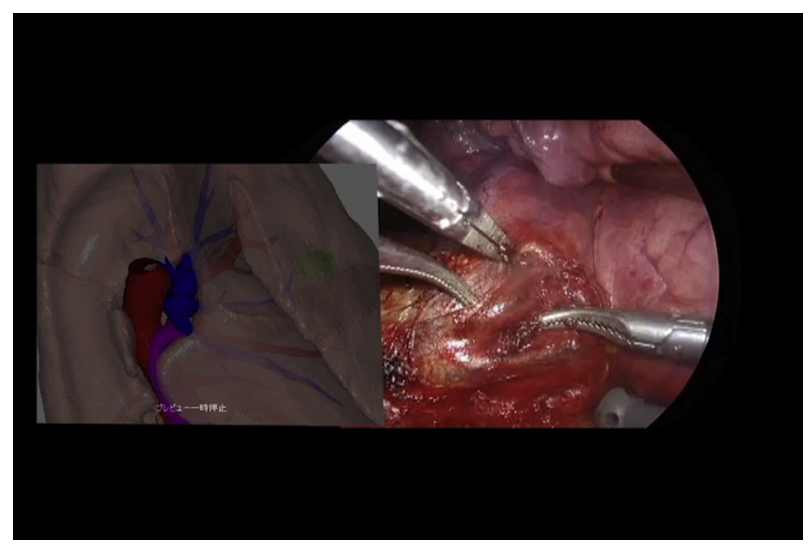

VIDEO 4. Comparison of the virtual dynamic image and the surgical video in a case undergoing left S3 segmentectomy. Virtual image is on the left side, and surgical video is on the right side. PV is in blue, PA is in red, and bronchi is in purple. First, a scene of dissecting the PV is shown. It was described similarly, and V3 was divided. The PA was identified. A3 and mediastinal A4 +5 and a tiny branch of A3 are described accurately. After cutting the PA, we opened the fissure, dissected the PA and the bronchi, encircled the B3, and divided it. In the virtual image, bronchi were described similarly. Video available at: https://www.jtcvs.org/article/ S0022-5223(19)31769-6/fulltext.

and to work smoothly. We believed that it was important for surgeons to obtain the virtual image and to simulate in the RPM without any delay. In this retrospective study, the median time to generate the virtual image was $121.3 \mathrm{sec}$ onds. This implies that approximately 2 minutes were needed after conventional 3D reconstruction, which would be widely performed in daily practice. The median of the calculation time, which was $15.3 \mathrm{~ms}$ showing approximately 65 frames in 1 second, demonstrated that virtual image worked smoothly.

For the data volume reduction and calculation time while working on the RPM, using STL data instead of Digital Imaging and Communications in Medicine data and centerline detection enabled simulation. In this system, STL data from any other CT reconstruction software would be available, and not only SYNAPSE VINCENT. This indicates the versatility of the RPM. The entire process is user-friendly because of its semi-automatic system, which allows surgeons to generate the virtual image by themselves without consulting a radiologist or technician. This also highlights the usefulness of the RPM.

The stakeholders of the RPM might include all levels of surgeons. Attending surgeons, who mainly tailor surgical strategies and train and educate novice surgeons, will benefit from this system, because it provides more detailed information based on patient-specific CT data. Moreover, the high-quality virtual images will help clinical fellows and residents understand the anatomy and preview the 


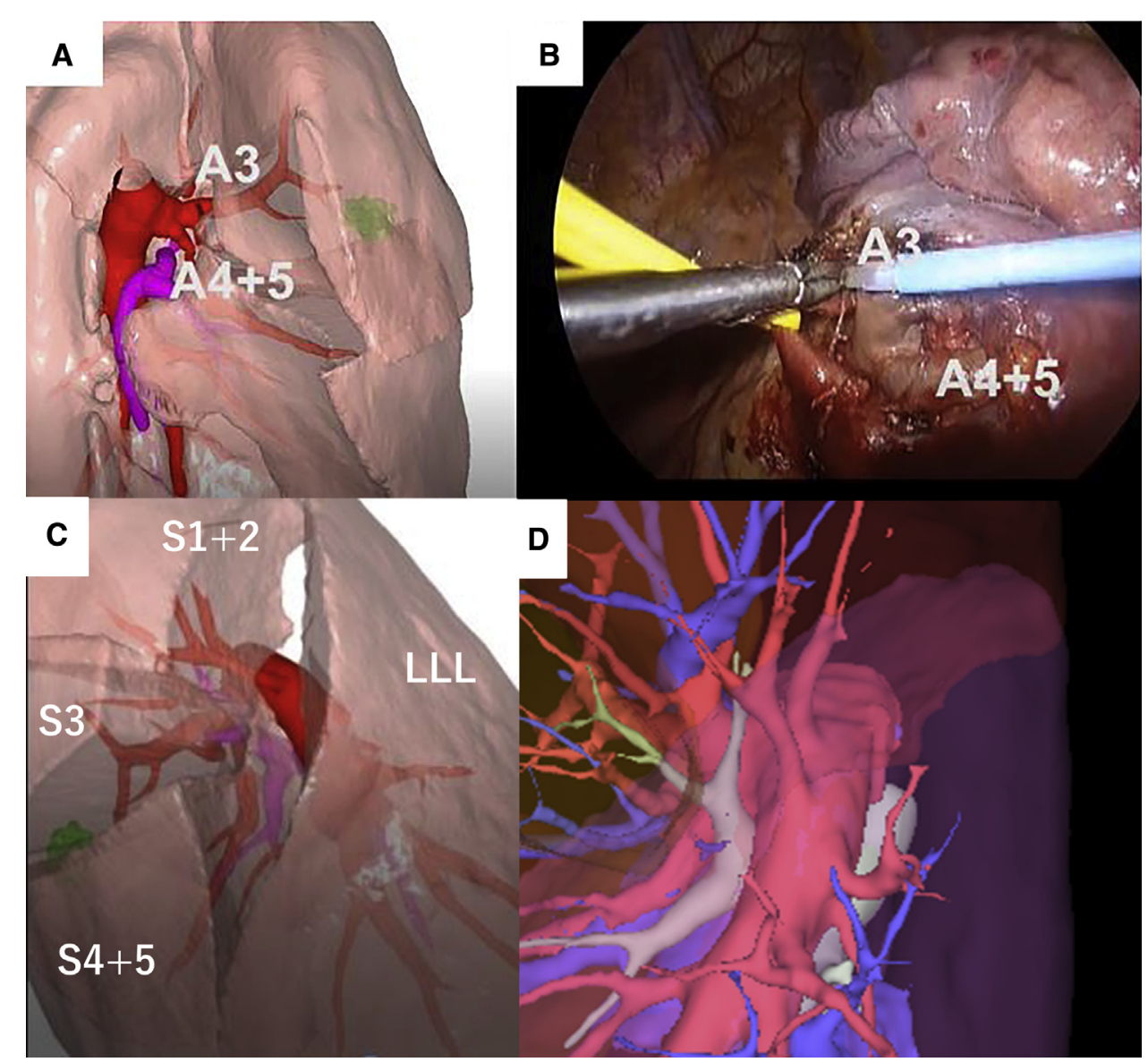

FIGURE 4. Representative case of left S3 segmentectomy comparing virtual image and surgical video. Virtual image was replicated by referring to surgical video. A, Snapshot of a dynamic image in the RPM during dissecting the hilum. B, Screen grab from the actual surgical video during dissecting the hilum. C, Snapshot of a dynamic image in the RPM during dissecting the fissure. D, Conventional 3D-CT. LLL, Left lower lobe.

surgery, and this system may be useful as an educational tool for students.

In this study, we validated the use of the RPM for pulmonary resection. Considering that the lung is the most deformable organ because of its softness, the RPM may be useful in the context of other organs, such as the liver and kidney. Because a high-quality simulation system is of paramount importance in the field of laparoscopic surgery, ${ }^{20,21}$ this novel system would be accepted in this field, too.

\section{Study Limitations}

Several limitations of this study need to be acknowledged. First, the system requires contrast-enhanced CT to generate the virtual dynamic image; however, this novel system, through 3D reconstruction CT in the SYNAPSE VINCENT, could build separate images of the PA, PV, and bronchi. ${ }^{2}$ Moreover, the accuracy of vessel descriptions or the bronchial tree in the RPM is totally dependent on the quality of contrast-enhanced CT and analysis in the SYNAPSE VINCENT. On occasion, we could not identify extremely tiny branches of the PA or PV through 3D reconstruction. ${ }^{4}$ Second, during actual surgery, the lung deforms not only because of manipulation or traction but also because of deflation; this is because in most cases, pulmonary resection is performed with 1-lung ventilation. Future studies are required to consider the deflation of the lung during surgery. Third, in this study, we developed a novel simulation system for pulmonary resection and validated it retrospectively in a small number of cases. In a future study, a multi-institutional study with a larger number of cases is required.

\section{CONCLUSIONS}

We developed a novel dynamic simulation system, the RPM, for anatomic pulmonary resection. The system generates virtual dynamic images from patient-specific CT. 


\section{Webcast}

You can watch a Webcast of this AATS meeting presentation by going to: https://aats.blob.core.windows.net/media/19\% 20AM/Sunday_May5/201DF/201DF/S46\%20-\%20Looking \%20to\%20the\%20future/S46_2_webcast_081019217.mp4.

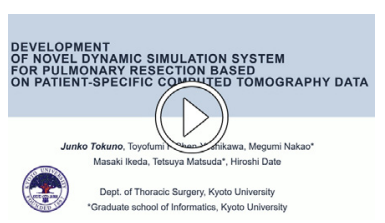

\section{Conflict of Interest Statement}

Authors have nothing to disclose with regard to commercial support.

\section{References}

1. Chen-Yoshikawa TF, Date H. Update on three-dimensional image reconstruction for preoperative simulation in thoracic surgery. $J$ Thorac Dis. 2016;8:S295-301.

2. Chen-Yoshikawa TF, Date H. Three-dimensional image in lung transplantation. Gen Thorac Cardiovasc Surg. 2018;6:19-26.

3. Kato H, Oizumi H, Suzuki J, Hamada A, Watarai H, Sadahiro M. Thoracoscopic anatomical lung segmentectomy using 3D computed tomography simulation without tumour markings for non-palpable and non-visualized small lung nodules. Interact Cardiovasc Thorac Surg. 2017;25:434-41.

4. Hagiwara M, Shimada Y, Kato Y, Nawa K, Makino Y, Furumoto H, et al. High-quality 3-dimensional image simulation for pulmonary lobectomy and segmentectomy: results of preoperative assessment of pulmonary vessels and short-term surgical outcomes in consecutive patients undergoing video-assisted thoracic surgery. Eur J Cardiothorac Surg. 2014;46:e120-6.

5. Fukuhara K, Akashi A, Nakane S, Tomita E. Preoperative assessment of the pulmonary artery by three-dimensional computed tomography before video-assisted thoracic surgery lobectomy. Eur J Cardiovasc Surg. 2008;34: 875-7.

6. Yokoyama Y, Sato M, Omasa M, Date H. Three-dimensional imaging for thoracoscopic resection of complex lung anomalies. Surg Case Rep. 2017;25: 106.

7. Solomon B, Bizekis C, Dellis SL, Donington JS, Oliker A, Balsam LB, et al. Simulating video-assisted thoracoscopic lobectomy: a virtual reality cognitive task simulation. J Thorac Cardiovasc Surg. 2011:141:249-55.

8. Jensen K, Bjerrum F, Hansen HJ, Petersen RH, Pedersen JH, Konge L, et al. A new possibility in thoracoscopic virtual reality simulation training: development and testing of a novel virtual reality simulator for video-assisted thoracoscopic surgery lobectomy. Interact Cardiovasc Thorac Surg. 2015;21:420-6.

9. Nakao M, Oda Y, Taura K, Minato K. Direct volume manipulation for visualizing intraoperative liver resection process. Comput Methods Programs Biomed. 2014; 113:725-35.

10. Nakao M, Taura K, Matsuda T. Resection process map for intraoperative cutting guides. Conf Proc IEEE Eng Med Biol Soc. 2016;2554-7.

11. Haouchine N, DequidtJ, Peterlik I, Kerrien E, Berger MO, Cotin S. Image-guided simulation of heterogeneous tissue deformation for augmented reality during hepatic surgery. Int Symp Mix Augment Real. 2013;199-208.

12. Nakao M, Minato K. Physics-based interactive volume manipulation for sharing surgical process. IEEE Trans Inf Technol Biomed. 2010;14:809-16.

13. Reynisson PJ, Scali M, Smistad E, Hofstad EF, Leira HO, Lindseth F, et al. Airway Segmentation and centerline extraction from thoracic CT - comparison of a new method to state of the art commercialized methods. PLoS One. 2015;12: e0144282.

14. Sironi A, Turetken E, Lepetit V, Fua P. Multiscale centerline detection. IEEE Trans Pattern Anal Mach Interll. 2016;7:1327-41.

15. Lidayová K, Frimmel H, Bengtsson E, Smedby Ö. Improved centerline tree detection of diseased peripheral arteries with a cascading algorithm for vascular segmentation. J Med Imaging. 2017;2:024004.
16. Werner R, Ehrhardt J, Schmidt R, Handels H. Modeling respiratory lung motion a biophysical approach using finite element methods. Int Soc Opt Eng. March 12, 2018; https://doi.org/10.1117/12.769155.

17. Novellis P, Bottoni E, Voulaz E, Cariboni U, Testori A, Bertolaccini L, et al. Robotic surgery, video-assisted thoracic surgery, and open surgery for early stage lung cancer: comparison of costs and outcomes at a single institute. $J$ Thorac Dis. 2018;10:790-8.

18. Cao C, Manganas C, Ang SC, Yan TD. A systematic review and meta-analysis on pulmonary resections by robotic video-assisted thoracic surgery. Ann Cardiothorac Surg. 2012;1:3-10.

19. Gondé H, Laurent M, Gillibert A, Sarsam OM, Varin R, Grimandi G, et al. The affordability of minimally invasive procedures in major lung resection: a prospective study. Interact Cardiovasc Thorac Surg. 2017;25:469-75.

20. Ashraf A, Collins D, Whelan M, O'Sullivan R, Balfe P. Three-dimensional (3D) simulation versus two-dimensional (2D) enhances surgical skills acquisition in standardized laparoscopic tasks: a before and after study. Int J Surg. 2015;14: 12-6.

21. Nepomnayshy D, Alseidi AA, Fitzgibbons SC, Stefanidis D. Identifying the need for and content of an advanced laparoscopic skills curriculum: results of a national survey. Am J Surg. 2016;211:421-5.

Key Words: surgery simulation, 3D reconstruction

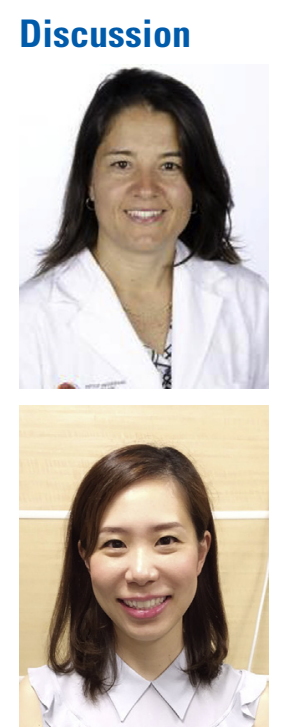

Dr Paula A. Ugalde (Quebec City, Quebec, Canada). This work is innovative and a great teaching tool. I have 3 questions for you. Is this software available in the market or is it exclusive through your service?

Dr Junko Tokuno (Kyoto, Japan). It is not available at this point, but we are thinking of making it a product in the near future, because we believe that it needs to be revised such as we would like to add the function describing intraoperative differentiation. So it needs to be revised.

Dr Ugalde. Perfect. My second question is, the system describes the anatomy perfectly with precision. Can it also measure the diameter of vessels, because now we are using more energy devices that are used according to the size of the vessels. Can you do that?

Dr Tokuno. It is a critically important question. The answer is yes, we can, but we haven't measured so far. It is interesting to see whether the vessels should be divided by an energy device. So according to your comment, we would like to measure from now on.

Dr Ugalde. So my last question on the same line, if it measures the diameter of the vessels, can we also measure the thickness of the parenchyma or the thickness of the bronchi to better define what type of cartridge we should use?

Dr Tokuno. For bronchi it is the same as the vessels; from now on, we would like to measure it. And for 
parenchyma, as I said before, we need to consider the differentiation. So in the near future it should be possible.

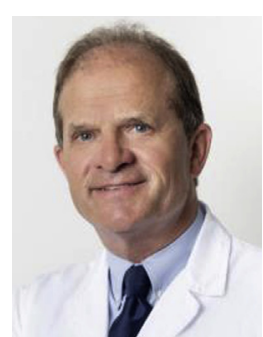

Dr Walter Weder (Zurich, Switzerland). The better we can plan surgery, the better is the quality, and I think this will help us. Can you also show us the lymph nodes? Usually when we have the vessel dissected it's not so difficult to get the overview, but the relationship with the lymph nodes is sometimes a problem. Can you make a 3D demonstration of the lymph nodes adjacent to the vessels?
Dr Tokuno. No. It could describe the location or existence of tumor or lymph node, but it can't show how severe the invasion to PA is. So it is one of the limitations.

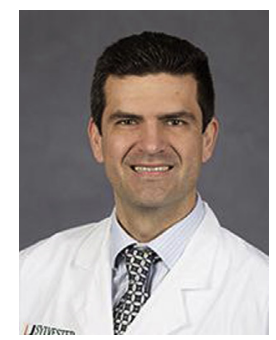

Dr Nestor Villamizar (Miami, Fla). Can this show us the intersegmental planes so we can see where the tumor is located in like the intersegmental fissure?

Dr Tokuno. Yes, we can. 